\title{
Closed manifolds admitting metrics with the same geodesics
}

\author{
Vladimir S. Matveev*
}

\begin{abstract}
The goal of this survey is to give a list of resent results about topology of manifolds admitting different metrics with the same geodesics. We emphasize the role of the theory of integrable systems in obtaining these results.
\end{abstract}

\section{Introduction}

\subsection{Definitions}

Definition 1 Let $g$ be a Riemannian metric on a manifold $M^{n}$ of dimension $n \geq 2$. A Riemannian metric $\bar{g}$ on $M^{n}$ is called geodesically equivalent to $g$, if every geodesic of $\bar{g}$, considered as an unparameterized curve, is a geodesic of $g$.

Trivial examples of geodesically equivalent metrics can be obtained by considering proportional metrics $g$ and $C \cdot g$, where $C$ is a positive constant.

Definition 2 A manifold $M^{n}$ is called geodesically rigid, if every two geodesically equivalent Riemannian metrics on $M^{n}$ are proportional.

In other words, on geodesically rigid manifolds, unparameterized geodesics define the metric (modulo multiplication by a constant).

\section{$1.2 \quad$ History}

The theory of geodesically equivalent metrics has a long and fascinating history that goes back to the works of Beltrami, Dini and Levi-Civita.

Beltrami 2] was the first to observe that two nonproportional metrics (even on closed manifolds) can have the same geodesics.

At the end of his paper 2, Beltrami formulated the problem of describing all geodesically equivalent metrics (for surfaces.) It is not clear from the text

\footnotetext{
* Mathematisches Institut, Universität Freiburg, 79104 Germany matveev@email.mathematik.uni-freiburg.de
} 
whether he assumed the local or the global description; actually, his motivation came from a certain problem of cartography, which requires the global setting. Nevertheless, partially because of strong results of Dini, Levi-Civita, Weyl, E. Cartan and Eisenhart, the theory of geodesically equivalent metrics was mostly a local geometry.

Locally, in a neighborhood of almost every point, a complete description of geodesically equivalent metrics has been given by Dini [13] for surfaces and Levi-Civita [18] for manifolds of arbitrary dimension. As a corollary of this description, one can show that, at least for dimensions two and three, every open manifold has non-proportional geodesically equivalent metrics.

Later, geodesically equivalent metrics were considered by Weyl, E. Cartan and Eisenhart. Weyl studied geodesically equivalent metrics on the tensor level and found a few tensor reformulations of geodesic equivalence. One of his most remarkable results is the construction [35] of the projective Weyl tensor: if two metrics are geodesically equivalent, then their projective Weyl tensors coincide. E. Cartan [11] studied geodesic equivalence on the level of affine connections. He introduced the so-called projective connection, which allows reconstruction of geodesics as unparameterized curves. In his book 14, Eisenhart systematically applied both methods and obtained a series of local results.

It is clear that the classics such as Lie [19, Painleve [30, Levi-Civita and Eisenhart understood well the connection between integrable systems and geodesically equivalent metrics. But they did not use it, probably because they mostly were interested in the local aspects of geodesically equivalent metrics.

Global aspects have been intensively studied since 50th, firstly by French (Lichnerowicz), Soviet (Rashevskii and Solodovnikov) and Japanese (Yano) geometry schools. But, probably because of the influence of earlier researcher, all known global results require fairly strong additional geometrical assumptions.

Roughly speaking, one takes some geometric assumption written in tensor form, combines it with one of the tensor reformulations of geodesic equivalence and deduces some new object with global geometric properties, see the surveys of Mikes [29] and Aminova [1].

Recently, it was found that integrable systems provides a very effective tool for understanding the topology of the manifolds admitting geodesically equivalent metrics. We describe the connection between geodesically equivalent metrics and integrable geodesic flows in Section 3.1 Roughly speaking, the existence of $\bar{g}$ geodesically equivalent to $g$ allows one to construct integrals for the geodesic flow of $g$, see Theorem 4 for details. If the metric $g$ and $\bar{g}$ are strictly-non-proportional, the geodesic flow of $g$ is Liouville-integrable.

\section{Resent results}

Theorem 1 ([27]) Let $M^{n}$ be a closed connected manifold. Suppose two nonproportional Riemannian metrics $g, \bar{g}$ on $M^{n}$ are geodesically equivalent. If the fundamental group of $M^{n}$ is infinite, then there exist $r \in\{1,2, \ldots, n-1\}$, a Riemannian metric $\tilde{g}$ and foliations $B_{r}$ (of dimension $r$ ) and $B_{n-r}$ (of dimension 
$n-r)$ such that, in a neighborhood $U(p)$ of every point $p \in M^{n}$, there exist coordinates

$$
(\bar{x}, \bar{y})=\left(\left(x_{1}, x_{2}, \ldots, x_{r}\right),\left(y_{r+1}, y_{r+2}, \ldots, y_{n}\right)\right)
$$

such that the $x$-coordinates are constant on every fiber of the foliation $B_{n-r} \cap$ $U(p)$, the $y$-coordinates are constant on every fiber of the foliation $B_{r} \cap U(p)$, and the metric $\tilde{g}$ has the block-diagonal form

$$
d s^{2}=\sum_{i, j=1}^{r} G_{i j}(\bar{x}) d x_{i} d x_{j}+\sum_{i, j=r+1}^{n} H_{i j}(\bar{y}) d y_{i} d y_{j},
$$

where the first block depends on the first $r$ coordinates and the second block depends on the remaining $n-r$ coordinates.

Theorem 1 already gives us the complete list of all geodesically rigid closed surfaces [21]: a closed connected surface is geodesically rigid if and only if its Euler characteristic is negative.

More precisely, a closed connected surface of negative Euler characteristic has infinite fundamental group, and admits no one-dimensional foliation, so it is geodesically rigid.

A closed connected surface of nonnegative Euler characteristic is not geodesically rigid: it is diffeomorphic to the sphere or the projective plane or the torus or the Klein bottle. Examples of nonproportional geodesically equivalent metrics on the sphere and on the projective plane were essentially constructed by Beltrami [2]. Since the geodesics of every flat metric are straight lines, every two flat metrics on the torus (or on the Klein bottle) are geodesically related (that is, there exists a diffeomorphism that takes the geodesics of the first metric to the geodesics of the second), so the torus or the Klein bottle are not geodesically rigid as well.

For dimension three, a direct corollary [27] of Theorem 1]is

Corollary 1 Suppose $M^{3}$ is a connected closed manifold. Suppose there exist nonproportional Riemannian metrics on $M^{3}$ that are geodesically equivalent. Then, modulo the Poincaré conjecture, $M^{3}$ is finitely covered by the sphere $S^{3}$ or by the product $F^{2} \times S^{1}$, where $F^{2}$ is a closed surface.

It appears that Corollary 1is true 25, 28, also without assuming the Poincaré conjecture:

Theorem 2 Let nonproportional Riemannian metrics $g$ and $\bar{g}$ be geodesically equivalent on a closed connected three-dimensional manifold $M^{3}$. Then the manifold is homeomorphic either to a lens space or to a Seifert manifold with zero Euler number. Every lens space and every Seifert manifold with zero Euler number admits geodesically equivalent metrics which are nonproportional.

Theorems 12 give us a complete list of closed connected manifolds of dimension two and three admitting nontrivial geodesic equivalence. It will be much 
more complicated to obtain such list in every dimension. But still, in every dimension $n \geq 2$, there exists infinitely many geodesically rigid manifolds [23, 27]:

Theorem 3 Every closed connected manifold admitting a Riemannian metric of negative sectional curvature is geodesically rigid.

Note that in view of result of Borel [10, in every dimension there exist infinitely many closed manifolds admitting metrics of negative sectional curvature.

\section{Methods and ideas}

\subsection{Integrability for the geodesic flows of geodesically equiv- alent metrics}

New methods for the global (= on closed or complete manifolds) investigation of geodesically equivalent metrics are based on the following observation 20, [21, [26]: the existence of $\bar{g}$ geodesically equivalent to $g$ allows one to construct commuting integrals for the geodesic flow of $g$.

Let $g=\left(g_{i j}\right)$ and $\bar{g}=\left(\bar{g}_{i j}\right)$ be Riemannian metrics on a manifold $M^{n}$. Consider the (1,1)-tensor $L$ given by the formula

$$
L_{j}^{i} \stackrel{\text { def }}{=}\left(\frac{\operatorname{det}(\bar{g})}{\operatorname{det}(g)}\right)^{\frac{1}{n+1}} \bar{g}^{i \alpha} g_{\alpha j} .
$$

Then, $L$ determines the family $S_{t}, t \in R$, of $(1,1)$-tensors

$$
S_{t} \stackrel{\text { def }}{=} \operatorname{det}(L-t \mathrm{Id})(L-t \mathrm{Id})^{-1} \text {. }
$$

Remark 1 Although $(L-t \mathrm{Id})^{-1}$ is not defined for $t$ lying in the spectrum of $L$, the tensor $S_{t}$ is well-defined for every $t$. Moreover, $S_{t}$ is a polynomial in $t$ of degree $n-1$ with coefficients being (1,1)-tensors.

We will identify the tangent and cotangent bundles of $M^{n}$ by $g$. This identification allows us to transfer the natural Poisson structure from $T^{*} M^{n}$ to $T M^{n}$.

Theorem 4 If $g, \bar{g}$ are geodesically equivalent, then, for every $t_{1}, t_{2} \in R$, the functions

$$
I_{t_{i}}: T M^{n} \rightarrow R, \quad I_{t_{i}}(v) \stackrel{\text { def }}{=} g\left(S_{t_{i}}(v), v\right)
$$

are commuting integrals for the geodesic flow of $g$.

In other direction these theorem is wrong; a counterexample could be found in $[22$. 
Theorem 5 (22]) Suppose for every $t \in R$ the function $I_{t}$ given by 4$)$ is an integral for the geodesic flow of $g$. If the Nijenhuis torsion $N_{L}$ vanishes, the metrics are geodesically equivalent.

Theorem 6 (9]) Let $g, \bar{g}$ be geodesically equivalent. Then the Nijenhuis torsion $N_{L}$ vanishes.

\subsection{What is special in these integrals?}

Definition 3 Two metrics $g$ and $\bar{g}$ are strictly-non-proportional at $x \in$ $M^{n}$, if all roots of $P(t):=\operatorname{det}(g-t \bar{g})$ are simple.

Let us assume that there exists a point where the metrics are strictly-nonproportional. Then, it is so 27 at almost every point, and the family $I_{t}$ contains $n$ integrals that are functionally independent almost everywhere. Hence, the geodesic flow of the metric is Liouville-integrable.

Let us note that

- the integrals are quadratic in velocities,

- at every point $x \in M^{n}$, the integrals (considered as quadratic forms) can be simultaneously diagonalizable.

Integrable systems with such properties [32] are known as Stäckel systems. Locally, in a given coordinate system, it can be defined by using a $(n \times n)$-matrix such that its columns depend on the corresponding coordinate only.

Not every stäckel system can come from geodesically equivalent metrics. The additional assumption is that the stäckel matrix can be chosen to be a Vandermonde matrix.

Stäckel systems with such property were also intensively studied. One of the reasons for it that they satisfy Robertson's [31] condition, which imply that its quantization is quantum-integrable as well. The second reasons is that all

stäckel systems coming from physics are of this type (or, a degeneration of systems of this type).

It is possible to show 22, that this extra-condition is also a sufficient condition for the existence of geodesically equivalent metrics.

The systems with this condition appear independently and under different names (L-systems, Benenti systems, quasi-bihamiltonian systems) in works of different authors [3, 4, 5, 6, 16, 12,

\subsection{If the metrics are strictly non-proportional}

Theorem 4 can be used most efficiently when there exists a point of a manifold where the metrics are strictly-non-proportional. Then, the geodesic flow of $g$ is Liouville-integrable, and we can apply the well-developed machinery of integrable systems. For example, the following theorem follows directly from Theorem 4 and Taimanov 33 . 
Theorem 7 Suppose $M^{n}$ is a connected closed manifold. Let the real-analytic Riemannian metrics $g$ and $\bar{g}$ on $M^{n}$ be geodesically equivalent. Suppose there exists a point of the manifold where the metrics are strictly-non-proportional. Then, the following statements hold.

1. The first Betti number $b_{1}\left(M^{n}\right)$ is not greater than $n$.

2. The fundamental group $\pi_{1}\left(M^{n}\right)$ is virtually Abelian.

The integrals are quadratic in velocities. Combining this fact with topological obstructions [17] for the existence of quadratically-integrable geodesic flows on closed surfaces, we obtain Theorem 3 for dimension two [21.

Note that, in view of results 34], Corollary 1 follows from Theorem 7 under the additional assumption that the metrics are real-analytic and that there exists a point where the metrics are strictly-non-proportional .

\subsection{Geodesic equivalence and zero entropy}

All results of this section are joint with Kruglikov; the proofs will be published elsewhere.

It is expected, that an integrable geodesic flow has zero topological entropy. This is not always the case. There are examples [7, 8] of integrable flows with non-zero entropy. But still it is possible to show that if the integrals come from strictly-non-proportional geodesically equivalent metrics by applying Theorem 4 the topological entropy of the geodesic flow must be zero.

Theorem 8 Suppose the Riemannian metrics $g, \bar{g}$ on a closed connected $M^{n}$ are geodesically equivalent. Suppose there exists a point where the metrics are strictly non-proportional. Then, the topological entropy of the geodesic flow of $g$ is zero.

Combining this theorem with the famous Yomdin's Theorem [15], we obtain

Corollary 2 Suppose the Riemannian metrics $g, \bar{g}$ on a closed connected $M^{n}$ are geodesically equivalent. Suppose there exists a point where the metrics are strictly non-proportional. Then, the manifold is finitely covered by the product of a rational-elliptic manifold and the torus.

\subsection{General case}

We will sketch the proof of Theorem 1 Consider the (1,1)-tensor $L$ given by the formula (2). All its eigenvalues are real. At every point $x \in M^{n}$, let us denote them by $\lambda_{1}(x) \leq \ldots \leq \lambda_{n}(x)$. It appears that they are globally ordered [26, 27, 28]:

Theorem 9 Let $\left(M^{n}, g\right)$ be a connected Riemannian manifold. Suppose every two points of the manifold can be connected by a geodesic. Let Riemannian metric $\bar{g}$ on $M^{n}$ be geodesically equivalent to $g$.

Then, for every $i \in\{1, \ldots, n-1\}$, for all $x, y \in M^{n}$, the following holds: 
1. $\lambda_{i}(x) \leq \lambda_{i+1}(y)$.

2. If $\lambda_{i}(x)<\lambda_{i+1}(x)$ for some $x \in M^{n}$, then $\lambda_{i}(z)<\lambda_{i+1}(z)$ for almost every point $z \in M^{n}$.

3. If $\lambda_{i}(x)=\lambda_{i+1}(y)$, then there exists $z \in M^{n}$ such that $\lambda_{i}(z)=\lambda_{i+1}(z)$.

Thus, if $g$ and $\bar{g}$ on closed connected $M^{n}$ are geodesically equivalent, then the following two cases are possible:

Case 1: There exists $r \in\{1, \ldots, n-1\}$ and a constant $\lambda \in \mathbb{R}$ such that, for every $x \in M^{n}$

$$
\lambda_{r}(x)<\lambda<\lambda_{r+1}(x) .
$$

Case 2: The following two conditions hold:

(i) For every $r \in\{1, \ldots, n-1\}$, the maximum $\max _{x \in M^{n}}\left(\lambda_{r}(x)\right)$ is equal to the minimum $\min _{x \in M^{n}}\left(\lambda_{r+1}(x)\right)$.

(ii) At least one of the eigenvalues of $L$ is not constant.

In the first case, it is possible to canonically construct [27] the metric $\tilde{g}$ and the foliations $B_{r}$ and $B_{n-r}$ as in Theorem 1

Let us explain where the foliations are coming from. At every point $x \in M^{n}$, let us denote by $V_{r}(x)\left(V_{n-r}(x)\right.$, respectively) the sum

$$
\bigoplus_{i=1}^{r} E_{\lambda_{i}} \quad\left(\bigoplus_{i=r+1}^{n} E_{\lambda_{i}}, \text { respectively }\right)
$$

where $E_{\lambda_{i}} \in T_{x} M^{n}$ is the eigenspace corresponding to $\lambda_{i}$.

Under the assumptions of Case $1, V_{r}$ and $V_{n-r}$ are smooth distributions of dimensions $r$ and $n-r$. By Theorem [6 they are integrable. Then, they generate two foliations $B_{r}$ and $B_{n-r}$.

The construction of the metric $\tilde{g}$ from Theorem $\square$ is based on the classical Levi-Civita's Theorem [18.

In the second case, it is possible to show that the fundamental group of the manifold is finite. The key instrument for it is Theorem 6 of the paper [27] which, roughly speaking, tells us that every (closed) manifold with two geodesically equivalent metrics satisfying $(i),(i i)$ has a closed submanifold $U$ with two geodesically equivalent metrics satisfying $(i),(i i)$ such that the natural homomorphism $\operatorname{Id}_{*}: \pi_{1}(U) \rightarrow \pi_{1}\left(M^{n}\right)$ is a surjection. Consequently applying this theorem, we come to one of the following subcases:

SC 1: The dimension $n$ of the manifold $M^{n}$ is $q+1$, where $q \geq 1$. The eigenvalues $\lambda_{1}=\ldots=\lambda_{q} \stackrel{\text { def }}{=} \lambda$ are constant, the eigenvalue $\lambda_{q+1}$ is not constant and there exists $z \in M^{q+1}$ such that $\lambda_{q+1}(z)=\lambda$.

SC 2: The dimension $n$ of the manifold $M^{n}$ is 2. The eigenvalues $\lambda_{1}$ and $\lambda_{2}$ are not constant and there exists a point $z \in M^{2}$ such that $\lambda_{1}(z)=\lambda_{2}(z)$. 
SC 3: The dimension $n$ of the manifold $M^{n}$ is $q+2$, where $q \geq 1$, the eigenvalues $\lambda_{1}$ and $\lambda_{q+2}$ are not constant and there exist $z_{1}, z_{2} \in M^{n}$ such that $\lambda_{1}\left(z_{1}\right)=\lambda_{q+2}\left(z_{2}\right)$.

SC 4: The dimension $n$ of the manifold $M^{n}$ is $n=q+r+1 ; q>0, r>0$. The eigenvalues $\lambda_{1}=\lambda_{2}=\ldots=\lambda_{r}$ and $\lambda_{r+2}=\lambda_{r+3}=\ldots=\lambda_{n}$ are constant. The eigenvalue $\lambda_{r+1}$ is not constant. There exist points $z_{0}, z_{1} \in M^{n}$ such that $\lambda_{r+1}\left(z_{0}\right)=\lambda_{1}$ and $\lambda_{r+1}\left(z_{1}\right)=\lambda_{n}$.

It is possible to show 27] that in all four subcases the fundamental group in finite.

\section{Acknowledgments}

In this paper I collected results of three years work; I am very grateful to many different people for their interest in this problem. Especially, I would like to thank W. Ballmann, V. Bangert, A. Bolsinov, K. Burns, A. Fomenko, M. Gromov, U. Hamenstädt, M. Igarashi, K. Kiyohara, B. Kruglikov, A. Naveira, P. Seidel, P. Topalov and K. Voss for fruitful discussions.

I would like to thank The University of Tromso (where the results of Section 3.4 were obtained) for the hospitality.

I also would like to thank The European Post-Doctoral Institute, The MaxPlanck Institute for Mathematics (Bonn) and The Isaac Newton Institute for Mathematical Sciences for hospitality and partial financial support. My research at INIMS has been supported by EPSRC grant GRK99015. My research was partially supported by DFG-programm 1154 (Global Differential Geometry) and Ministerium für Wissenschaft, Forschung und Kunst Baden-Württemberg (Eliteförderprogramm Postdocs 2003).

\section{References}

[1] A. V. Aminova, Projective transformations of pseudo-Riemannian manifolds. Geometry, 9. J. Math. Sci. (N. Y.) 113(2003), no. 3, 367-470.

[2] E. Beltrami, Resoluzione del problema: riportari i punti di una superficie sopra un piano in modo che le linee geodetische vengano rappresentante da linee rette, Ann. Mat., 1(1865), no. 7, 185-204.

[3] S. Benenti, Inertia tensors and Stäckel systems in the Euclidean spaces, Differential geometry (Turin, 1992). Rend. Sem. Mat. Univ. Politec. Torino 50(1992), no. 4, 315-341.

[4] S. Benenti, Orthogonal separable dynamical systems, Differential geometry and its applications (Opava, 1992), 163-184, Math. Publ., 1, Silesian Univ. Opava, Opava, 1993. 
[5] S. Benenti, An outline of the geometrical theory of the separation of variables in the Hamilton-Jacobi and Schrödinger equations, SPT 2002: Symmetry and perturbation theory (Cala Gonone), 10-17, World Sci. Publishing, River Edge, NJ, 2002.

[6] S. Benenti, Special symmetric two-tensors, equivalent dynamical systems,cofactor and bi-cofactor systems, preprint.

[7] A.V. Bolsinov, I.A. Taumanov, An example of an integrable geodesic flow with positive topological entropy, Russian Math. Surveys 54(1999), no. 4, 833-834.

[8] A.V. Bolsinov, I.A. Taŭmanov, Integrable geodesic flows with positive topological entropy, Invent. Math. 140(2000), no. 3, 639-650.

[9] Alexei V. Bolsinov, Vladimir S. Matveev, Geometrical interpretation of Benenti's systems, J. of Geometry and Physics, 44(2003) 489-506.

[10] A. Borel, Compact Clifford-Klein forms of symmetric spaces, Topology 2(1963) 111-122.

[11] E. Cartan, Lecons sur la theorie des espaces a connexion projective. Redigees par P. Vincensini, Paris: Gauthier-Villars., 1937.

[12] M. Crampin, W. Sarlet, G. Thompson, Bi-differential calculi, biHamiltonian systems and conformal Killing tensors, J. Phys. A 33(2000), no. $48,8755-8770$.

[13] U. Dini, Sopra un problema che si presenta nella theoria generale delle rappresetazioni geografice di una superficie su un'altra, Ann. Mat., ser.2, 3(1869), 269-293.

[14] L. P. Eisenhart, Riemannian Geometry. 2d printing, Princeton University Press, Princeton, N. J., 1949.

[15] M. Gromov, Entropy, homology and semialgebraic geometry, Séminaire Bourbaki, 1985/86. Astrisque No. 145-146(1987), 5, 225-240.

[16] A. Ibort, F. Magri, G. Marmo, Bihamiltonian structures and Stäckel separability, J. Geom. Phys. 33 (2000), no. 3-4, 210-228.

[17] V. N. Kolokol'tzov, Geodesic flows on two-dimensional manifolds with an additional first integral that is polynomial with respect to velocities, Math. USSR-Izv. 21(1983), no. 2, 291-306.

[18] T. Levi-Civita, Sulle trasformazioni delle equazioni dinamiche, Ann. di Mat., serie $2^{a}, \mathbf{2 4}(1896), 255-300$.

[19] S. Lie, Untersuchungen über geodätische Kurven, Math. Ann. 20(1882). Can be found in Sophus Lie Gesammelte Abhandlungen, Band 2, erster Teil, 267-374. Teubner, Leipzig 1935. 
[20] V. S. Matveev, P. J. Topalov, Trajectory equivalence and corresponding integrals, Regular and Chaotic Dynamics, 3(1998), no. 2, 30-45.

[21] V. S. Matveev and P. J. Topalov, Metric with ergodic geodesic flow is completely determined by unparameterized geodesics, Electron. Res. Announc. Amer. Math. Soc., 6(2000) 98-104.

[22] V. S. Matveev, P. J. Topalov, Quantum integrability for the BeltramiLaplace operator as geodesic equivalence, Math. Z. 238(2001) 833-866.

[23] Vladimir S. Matveev, Geschlossene hyperbolische 3-Mannigfaltigkeiten sind geodätisch starr, Manuscripta Math. 105(2001), no. 3, 343-352.

[24] Vladimir S. Matveev, Low-dimensional manifolds admitting metrics with the same geodesics, Contemp. Math., 308(2002), 229-243.

[25] Vladimir S. Matveev, Three-manifolds admitting metrics with the same geodesics, Math. Research Letters, 9(2002), no. 2-3, 267-276.

[26] Peter J. Topalov, Vladimir S. Matveev, Geodesic equivalence via integrability, Geometriae Dedicata 96(2003) 91-115.

[27] Vladimir S. Matveev, Hyperbolic manifolds are geodesically rigid, Invent. math. 151(2003) 579-609.

[28] Vladimir S. Matveev, Three-dimensional manifolds having metrics with the same geodesics, Topology 42(2003) no. 6, 1371-1395.

[29] J. Mikes, Geodesic mappings of affine-connected and Riemannian spaces. Geometry, 2., J. Math. Sci. 78(1996), no. 3, 311-333.

[30] P. Painlevé, Sur les intégrale quadratiques des équations de la Dynamique, Compt.Rend., 124(1897) 221-224.

[31] H. P. Robertson, Bemerkung über separierbare Systeme in der Wellenmechanik, Math. Ann. 98(1927) 749-752.

[32] P. Stäckel, Integration der Hamilton-Jacobischen Differentialgleichungen mittels Separation der Variabeln, Habilitationsschrift, Halle, 1891.

[33] I. A. Taimanov, Topological obstructions to the integrability of geodesic flow on nonsimply connected manifold, Math.USSR-Izv., 30(1988), no. 2, 403409.

[34] F. Waldhausen, Gruppen mit Zentrum und 3-dimensionale Mannigfaltigkeiten, Topology 6(1967) 505-517.

[35] H. Weyl, Zur Infinitisimalgeometrie: Einordnung der projectiven und der konformen Auffasung, Nachrichten von der K. Gesellschaft der Wissenschaften zu Göttingen, Mathematisch-Physikalische Klasse; "Selecta Hermann Weyl", Birkhäuser Verlag, Basel und Stuttgart, 1956. 